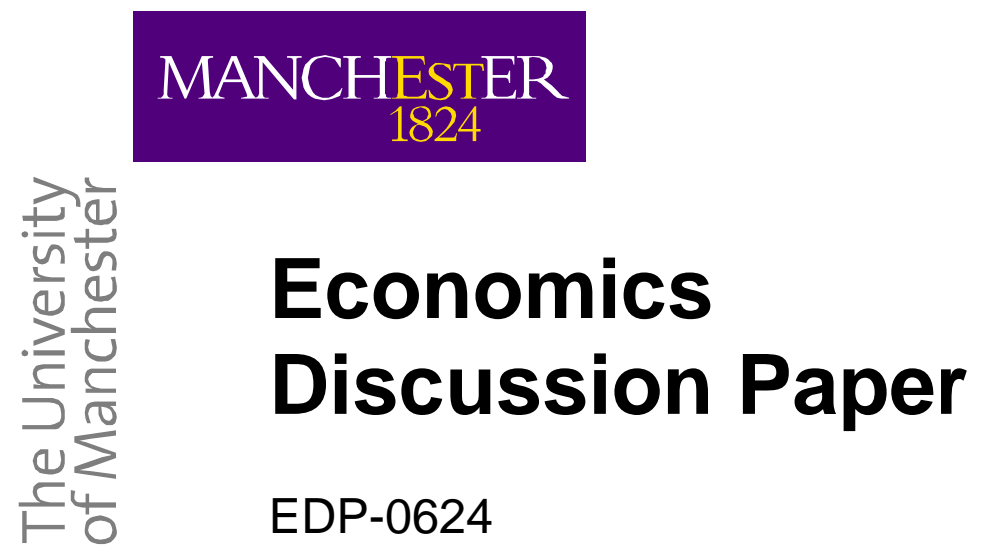

\title{
Weak Links, Good Shots and other Public Good Games: Building on BBV
}

\author{
Richard Cornes and Roger Hartley
}

September 2006 


\title{
Weak Links, Good Shots and other Public Good Games: Building on BBV
}

\author{
Richard Cornes \\ School of Economics \\ University of Nottingham \\ University Park \\ Nottingham \\ NG7 2RD \\ UK \\ rccornes@aol.com \\ Roger Hartley \\ Economic Studies \\ School of Social Sciences \\ University of Manchester \\ Oxford Road, Manchester \\ M13 9PL \\ UK \\ roger.hartley@manchester.ac.uk
}

August 15, 2006 


\begin{abstract}
We suggest an alternative way of analyzing the canonical Bergstrom-BlumeVarian model of non-cooperative voluntary contributions to a public good that avoids the proliferation of dimensions as the number of players is increased. We exploit this approach to analyze models in which the aggregate level of public good is determined as a more general social composition function of individual gifts - specifically, as a generalized CES form - rather than as an unweighted sum as well as the the weakest-link and best-shot models suggested by Hirshleifer. In each case, we characterize the set of equilibria, in some cases establishing existence of a unique equilibrium as well as briefly pointing out some interesting comparative static properties. We also study the weakest-link and best-shot limits of the CES composition function and show how the former can be used for equilibrium selection and the latter to show that equilibria of some better-shot games are identical to those of the much simpler best-shot game.
\end{abstract}

Keywords: noncooperative games, public goods, weakest links, best shots. JEL classifications: C72, H41. 


\section{Introduction}

The canonical model of non-cooperative public good provision set out by Bergstrom, Blume and Varian [2] - hereafter BBV - is a prominent example of a noncooperative game with an aggregative structure. The common object of all players' preferences is a simple aggregate, the unweighted sum of all individual contributions $g_{i}$, and each player's preferences can be represented by a payoff function of the form $\pi_{i}\left(g_{i}, G\right)$, where $G=\sum_{j} g_{j}$.

We study a systematic way of exploiting its aggregative structure that avoids the proliferation of dimensions as the number of players grows and thereby simplifies its analysis. This permits us to extend the model by allowing $G$ to be a more general (social composition) function of individual contributions, rather than an unweighted sum. This extra generality allows us to consider weaker link and better shot situations, of which Hirshleifer's [7] weakest link and best shot games are extreme cases. Social composition functions involving weaker-link public goods exhibit a convex technology for transforming individual contributions into the aggregate level of $G$, and imply a unique equilibrium in pure strategies. Situations involving better-shot public goods have non-convex social composition functions and, typically, multiple equilibria. Our approach also elucidates structural properties of equilibria and comparative statics properties. To avoid excessive length, we avoid a full treatment of these topics, confining ourselves to passing remarks.

In Sections 2 and 3, we show how "replacement functions" offer a simple and intuitive proof of existence, uniqueness and neutrality in the canonical model of BBV. The main aim of these sections is to set the scene for extensions of the canonical model which yield to modifications of this approach. For example, in Section 4, we show how easily an appropriate redefinition of replacement functions shows that Hirshleifer's weakest-link model has a continuum of Pareto ranked equilibria. A more radical extension of the replacement function can be used to analyze games in with a generalized concave CES social composition function. In Section 5, we prove that such games always have a unique equilibrium. The fact that the weakest link composition function can be viewed as a limiting case of CES suggests using the limiting equilibrium to select from the continuum of equilibria under weakest link. We show that this can indeed be done, but it does not typically select the Pareto dominant equilibrium. A further extension of replacement functions (to correspondences) allows us to handle non-concave social composition functions and we illustrate this by discussing games with convex CES composition functions and Cobb-Douglas preferences in Section 6. When the (negative) elasticity of substitution is close enough to zero, we offer a complete characterization of the set of equilibria. In particular, 
we characterize all possible sets of players active in some equilibrium and show that, given such a set, the equilibrium is uniquely determined. A final extension (to "upper" replacement functions) allows us to characterize the set of equilibria in best-shot games and to show that Pareto-improving and coordination-resolving transfers may be available in such games. Finally, we show that when the elasticity of substitution is negative and close enough to zero in the better-shot game, the set of equilibria coincides with that in the best-shot game.

\section{The Canonical Model}

We generally follow BBV's notation and assumptions. Their model has four elements:

1. Individual Preferences Player $i, i=1, \ldots, n$, has preferences represented by the utility function $u_{i}\left(x_{i}, G\right)$, where $x_{i} \geq 0$ is the quantity of a private good and $G$ the total quantity of a pure public good. BBV do not explicitly impose much structure directly on preferences. In particular, they assume that both goods are desirable and strictly normal. For convenience, we shall assume, without explicit statement, that $u_{i}$ is continuously differentiable. Then, binormality implies that marginal rates of substitution are is strictly decreasing in $G$ and non-decreasing in $g_{i}$. Note that increasing, strictly binormal preferences preferences are also strictly convex.

2. Individual Budget Constraints Player $i$ 's budget constraint requires that

$$
x_{i}+g_{i}=w_{i}
$$

where $g_{i} \geqq 0$ is her contribution to a pure public good, or her gift, and $w_{i}$ is her exogenous income.

3. The Social Composition Function The total public good provision is the unweighted sum of individual gifts ${ }^{1}$ :

$$
G=\sum_{i=1}^{n} g_{i}
$$

4. The Behavioral Assumption The game is a static, or simultaneous, noncooperative game in which the strategic choice variables are the individual gifts, $\left(g_{1}, g_{2}, \ldots, g_{n}\right)$.

\footnotetext{
${ }^{1}$ The term "social composition function" was suggested by Hirshleifer [7].
} 
BBV demonstrate the existence of a unique Nash equilibrium in pure strategies. Existence is established by appealing to Brouwer's fixed point theorem, and uniqueness by an argument tailored to the public good model. Although there is no doubting its formal correctness, their uniqueness argument has not struck all readers as intuitively transparent ${ }^{2}$.

Their comparative static analysis proceeds by direct examination of the budget set, and establishes the well-known neutrality result associated with income redistributions amongst contributors, as well as the limits on redistributions that maintain neutrality.

\section{An Alternative Approach: The Replace- ment Function}

Cornes and Hartley [5] formally demonstrate the existence, and explore the properties, of the replacement function $r_{i}$ of Player $i$, which expresses the player's best response as a function, not of the sum of best responses of all other players, but of the total level of public good $G$. That is, $g_{i}=r_{i}(G)$ if and only if the strategy choice of Player $i$ is $g_{i}$ in all Nash equilibria in which aggregate public good provision is $G$. (This includes the player's own choice amongst the arguments of the function.) They use replacement functions to provide simple analyses of existence, uniqueness and comparative static properties of equilibrium in simple public good models.

We now briefly sketch a derivation of replacement functions and their properties from first principles. This proves a useful baseline for subsequent discussions.

Denote player $i$ 's marginal rates of substitution ${ }^{3}$ by $M R S_{i}=\frac{\partial U_{i}}{\partial G} / \frac{\partial U_{i}}{\partial x_{i}}$. Then, taking $g_{i}$ and $G$ as arguments, her best response satisfies precisely one of the following conditions:

$$
\begin{aligned}
& M R S_{i}\left(\widehat{g}_{i}, G\right)=1 \text { and } 0 \leq \widehat{g}_{i} \leq w_{i} \\
& \operatorname{MRS}_{i}\left(\widehat{g}_{i}, G\right)>1 \text { and } \widehat{g}_{i}=w_{i} \\
& \operatorname{MRS} S_{i}\left(\widehat{g}_{i}, G\right)<1 \text { and } \widehat{g}_{i}=0
\end{aligned}
$$

We first establish that there is at most a single best reply by player $i$, $\widehat{g}_{i}$, consistent with a given value of $G$. To see this, fix $G$. An increase in $g_{i}$

\footnotetext{
${ }^{2}$ Indeed, in [3] they tighten up their original uniqueness argument in response to concerns voiced by Fraser [6].

${ }^{3}$ Note that our definition of $M R S_{i}$ presupposes differentiability of $u_{i}($.$) . This assump-$ tion can be dispensed with in much of what follows, and replaced with statements about the support of $u_{i}($.$) .$
} 


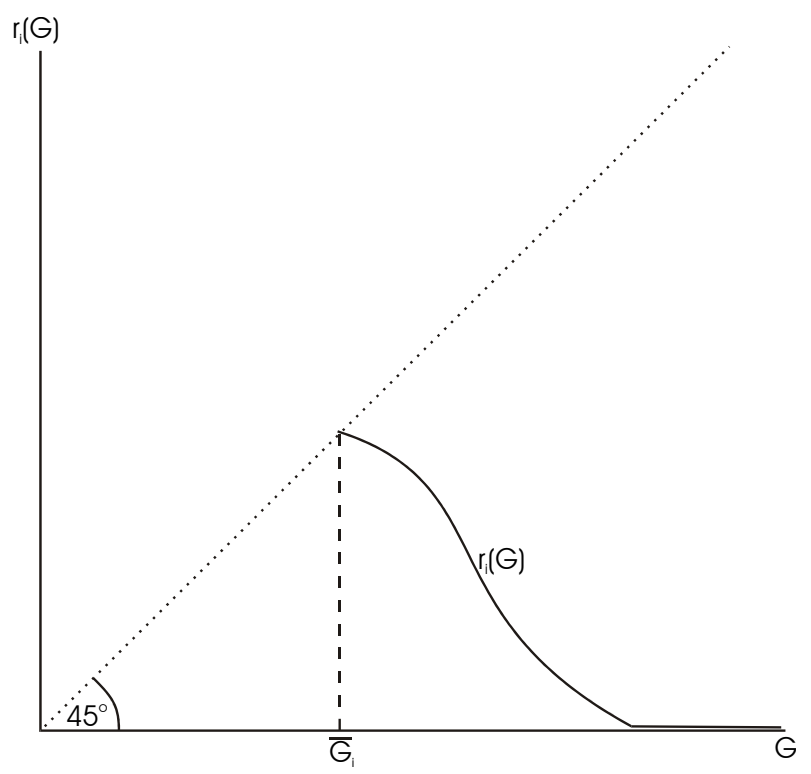

Figure 1:

implies a reduction in $x_{i}$ which, because of normality, implies a reduction in $M R S_{i}$. Hence $M R S_{i}\left(g_{i}, G\right)$ is strictly decreasing in $g_{i}$ and there is a unique $\widehat{g}_{i}$ satisfying one of the three conditions above.

Now consider an increase in $G$. For any given value of $g_{i}$, an increase in $G$ implies, again through normality, a lower value of $M R S_{i}$. Hence, an increase in $G$ shifts the graph of $M R S_{i}$ against $g_{i}$ downwards. This implies that, if initially $w_{i}>\widehat{g}_{i}>0$, then $\widehat{g}_{i}$ decreases in response to the increase in $G$.

Figure 1 graphs the replacement function whose existence and monotonicity have been established. It also shows several further properties that we have not explicitly discussed.

Cornes and Hartley [5] treat these matters more fully. For present purposes, the following observations are sufficient:

Proposition 3.1 In the canonical public good model, player $i$ has a replacement function $r_{i}(G)$ with the following properties:

1. There exists a finite value, $\bar{G}_{i}$, at which $r_{i}\left(\bar{G}_{i}\right)=\bar{G}_{i}$.

2. $r_{i}(G)$ is defined for all $G \geqq \bar{G}_{i}$.

3. $r_{i}(G)$ is continuous. 
4. $r_{i}(G)$ is everywhere non-increasing in $G$, and is strictly decreasing wherever it is strictly positive.

Here, $\bar{G}_{i}$ is the level of the public good that Player $i$ would prefer, if that player were the sole contributor. Replacement functions can be used to study equilibria via the following characterization, whose proof is trivial.

Characterization of a Nash equilibrium A strategy profile $\widehat{\mathbf{g}}$ is a Nash equilibrium if and only if

$$
\widehat{g}_{j}=r_{j}(\widehat{G}) \text { for } j=1, \ldots, n,
$$

where $\widehat{G}=\sum_{j=1}^{n} \widehat{g}_{j}$.

Note that $\widehat{G}$ is an equilibrium level of the public good if and only if it is a fixed point of the aggregate replacement function $R(G)=\sum_{j=1}^{n} r_{j}(G)$. It follows that, if $R$ has a unique fixed point, the game has a unique equilibrium. This will happen if $R$ is continuous, strictly decreasing where positive and has a graph that crosses the $45^{\circ}$ line. Exactly these properties follow from Proposition 3.1 which establishes the following result.

Theorem 3.1 In the canonical model, there exists a unique Nash equilibrium in pure strategies.

Not only do existence and uniqueness follow directly from simple geometric considerations. So, too, do other properties of equilibrium, including comparative static responses. We draw attention to two such results. Knowledge of the aggregate $G$ uniquely determines the choice of private good consumption of a positive contributor - this is determined as the point on the income expansion path corresponding to the given value of $G$. The following theorem follows immediately:

Theorem 3.2 Let players $i$ and $j$ be positive contributors at equilibrium in the canonical model. Suppose further that they have identical preferences. Then their equilibrium consumption bundles and utility levels are identical, even if their initial incomes differ.

The second property is that of neutrality. At a given value of $G$, a change in a contributors's income brings about an equal change in her level of contribution, as long as she remains at an interior solution:

$$
\widehat{g}_{i}=w_{i}-\widehat{x}_{i}(G)
$$




$$
\Longrightarrow \Delta \widehat{g}_{i}=\Delta w_{i} .
$$

It follows immediately that a transfer between two players who make positive contributions both before and after the transfer leaves the equilibrium allocation unaffected. The upward shift in one replacement function precisely cancels out the downward shift in the other, leaving the graph of the aggregate replacement function unaffected in the neighborhood of equilibrium. This gives the neutrality property:

Theorem 3.3 In the canonical model, a pure redistribution of income among a set of positive contributors that leaves that set unchanged has no effect on the equilibrium allocation.

Before our extension of the model in the following section, we introduce an alternative function, and an alternative way of characterizing a Nash equilibrium. This proves more convenient than the replacement function in certain settings. Dividing both sides by $G$, the equilibrium characterization becomes: $\widehat{G}$ is an equilibrium level of the public good if and only if

$$
S(\widehat{G})=\sum_{j=1}^{n} s_{j}(\widehat{G})=1,
$$

where $s_{i}(G)=r_{i}(G) / G$ is the share function of Player $i$ and $S(G)$ is the aggregate share function. The equilibrium strategy of Player $i$ is $\widehat{g}_{i}=\widehat{G} s_{i}(\widehat{G})$.

The advantage of using share functions is that a decreasing aggregate share function is sufficient for uniqueness and, although a decreasing aggregate replacement function entails this property, it is possible for share functions to be decreasing even where replacement functions are increasing or constant.

\section{Weakest-link Public Goods}

The approach of the previous section can be adapted to Hirshleifer's weakestlink and best-shot public good games. In this section, we discuss the weakest link social composition function (SCF):

$$
G=\min _{j=1, \ldots, n} g_{j}
$$

deferring treatment of the best-shot case to Section 7. We approach equilibria through the replacement function $r_{i}$ of Player $i$, where $r_{i}(G)$ is the 
unique strategy of the player in all equilibria in which aggregate level of the public good is $G$.

Let $\bar{G}_{i}$ denote the preferred level of the public good of Player $i$ as sole contributor and $G_{-i}=\min _{j \neq i} g_{j}$. For all contribution levels such that $G_{-i} \leq$ $\bar{G}_{i}$, Player $i$ will want to match the smallest of the other contributions, since her preferences are convex. In this event, her best response is $\widehat{g}_{i}=G_{-i}$ and the total quantity of the public good is $G=\min \left\{\widehat{g}_{i}, G_{-i}\right\}=\widehat{g}_{i}=G_{-i}$. However, if $G_{-i}>\bar{G}_{i}$, Player $i$ will only want to contribute up to the level $\bar{G}_{i}$, and no further. His contribution then determines the value of the weakest link. In this event, $G=\min \left\{\widehat{g}_{i}, G_{-i}\right\}=\bar{G}_{i}$. It follows that

$$
\left(r_{i}(G), G\right)=\left(G_{-i}, G_{-i}\right) \text { if } 0 \leq G_{-i} \leq \bar{G}_{i}
$$

and

$$
\left(r_{i}(G), G\right)=\left(\bar{G}_{i}, \bar{G}_{i}\right) \text { if } G_{-i}>\bar{G}_{i} .
$$

Proposition 4.1 If Player $i$ has convex increasing preferences, her replacement function has domain $\left[0, \bar{G}_{i}\right]$ and satisfies $r_{i}(G)=G$ in this domain.

Nash equilibrium levels of the public good are still fixed points of the aggregate replacement function $R$, provided the definition of $R$ is modified to $R(G)=\min _{j=1, \ldots, n} r_{j}(G)$. The domain of $R$ is the intersection of the domains of individual replacement functions, so

$$
R(G)=G \text { for } 0 \leq G \leq \min _{j=1, \ldots, n} \bar{G}_{i} .
$$

We may conclude that any non-negative level of the public good not exceeding any individually preferred level is an equilibrium.

Theorem 4.1 If all players have convex, increasing preferences, $\widehat{\mathrm{g}}$ is an equilibrium strategy profile if and only if $\widehat{g}_{j}=\widehat{g}$ for all $j$ for some $\widehat{g}$ satisfying $0 \leq \widehat{g} \leq \min _{j=1, \ldots, n} \bar{G}_{i}$.

Thus, there is a continuum of Pareto ranked equilibria. Introspection has suggested to students of this model that the salient equilibrium is precisely the value $\widehat{g}=\min _{j=1, \ldots, n} \bar{G}_{i}$, which Pareto-dominates all the others. Experimental evidence has not offered unqualified support to Pareto dominance as a selection criterion ${ }^{4}$.

Vicary and Sandler [16], [13] and [17] explore weakest-link games in which players are able to make income transfers.

\footnotetext{
${ }^{4}$ In Section 5.4, we will investigate explictly the limiting properties of the model as $\rho \longrightarrow-\infty$.
} 


\section{More General Concave Social Composition Functions}

\subsection{Properties of the social composition function}

In this and the next sections, we revisit the extension of the canonical model suggested by Cornes (1993) focussing on CES social composition functions. Indeed, in this section, we analyze a generalization of this SCF, which takes the following form:

$$
G=\left(\sum_{j=1}^{n} \beta_{j} g_{j}^{\rho_{j}}\right)^{1 / \rho},
$$

where $\beta_{1}, \ldots, \beta_{n}>0$. In this section, we use share functions to show that, under parameter restrictions implying that the SCF is concave, the voluntary provision game has a unique equilibrium. In the next section we analyze convex CES SCFs, where it is necessary to extend the concepts of replacement and share functions to correspondences.

Throughout this section we consider two distinct parameter regimes that imply a concave social composition function.

Parameter Set 1 We have

$$
0<\rho_{i} \leq \rho \leq 1 \text { and for } i=1, \ldots, n \text { and } \max _{j=1, \ldots, n} \rho_{j}<1 .
$$

The left-hand inequality excludes $\rho=0$. Of course, if $\rho_{i}=\rho$ for all $i$ and $\rho \longrightarrow 0$, the function (1) approaches Cobb-Douglas (with parameters $\beta_{i} / \sum_{j=1}^{n} \beta_{j}$ ). We do not explicitly study this case, but the principal qualitative conclusions (existence and uniqueness of an equilibrium) are the same as those under Parameter Set 1.

Parameter Set 2 We have

$$
\rho \leq \rho_{i}<0 \text { for } i=1, \ldots, n \text {. }
$$

Since all $\rho_{i}<0$ in Parameter Set 2, we cannot use (1) to calculate $G$ when some $g_{i}=0$; in this case we simply set $G=0$, to ensure continuity.

Under either parameter set, $G$ is a strictly increasing function of each $g_{i}$. Both parameter sets obviously include a CES SCF as a special case. However, Parameter Set 1 also includes, for example, a sum of constant elasticity production functions: $\sum_{j=1}^{n} \beta_{j} g_{j}^{\rho_{j}}$. We will show that, under both 
parameter sets, a unique equilibrium exists. However, the two sets imply interestingly contrasting comparative static responses.

In our analysis, it will prove convenient to use the facts that

$$
\frac{\partial G}{\partial g_{i}}=\frac{\beta_{i} \rho_{i} g_{i}^{\rho_{i}-1}}{\rho G^{\rho-1}}
$$

and, when (3) holds,

$$
\frac{\partial^{2} G}{\partial g_{i}^{2}}=-\frac{\beta_{i} \rho_{i}^{2}}{\rho} \frac{g_{i}^{\rho_{i}-2}}{G^{2 \rho-1}}\left\{\left(\frac{1}{\rho_{i}}-1\right) G^{\rho}-\left(\frac{1}{\rho}-1\right) \beta_{i} g_{i}^{\rho_{i}}\right\}
$$

using (3) to obtain the second line. We can use the inequality $G^{\rho} \geq \beta_{i} g_{i}^{\rho_{i}} \geq 0$ (a consequence of $\mathbf{g}_{-i} \geq \mathbf{0}$ ) to deduce the following lemma:

Lemma 5.1 Marginal $S C F: \partial G / \partial g_{i}$ is non-increasing in $g_{i}$ and strictly decreasing unless we have Parameter Set 1 with $\rho_{i}=\rho$ and $\mathbf{g}_{-i}=\mathbf{0}$.

\subsection{Preferences}

We maintain the assumptions of increasing preferences and binormality as in the canonical model of BBV. We find it convenient to write player $i$ 's payoff function of strategy profile $\mathbf{g}=\left(g_{1}, \ldots, g_{n}\right)$ as $\pi_{i}(\mathbf{g})=u_{i}\left(w_{i}-g_{i}, G\right)$.

\subsection{Best responses, replacement and share functions}

In this subsection, we show that under binormality and Parameter Sets 1 or 2 , replacement functions and therefore share functions exist. We also show that share functions are monotonic in $G$.

\subsubsection{Characterizing best responses}

The marginal payoff of Player $i$ (holding $\mathbf{g}_{-i}$ fixed) can be written

$$
\frac{\partial u_{i}}{\partial x_{i}} \frac{\partial G}{\partial g_{i}}\left\{M R S_{i}\left(w_{i}-g_{i}, G\right)-\left(\frac{\partial G}{\partial g_{i}}\right)^{-1}\right\} .
$$

Both terms outside the braces are positive (by binormality and (3)), so $\partial \pi_{i} / \partial g_{i}$ has the same sign as the term in braces. Furthermore, an increase in $g_{i}$ leads to an increase in $G$ and therefore a decrease in $w_{i}-g_{i}$ and, by binormality, a strict decrease in $M R S_{i}$. Lemma 5.1 shows that an increase in $g_{i}$ cannot cause an increase in $\partial G / \partial g_{i}$ and therefore $-\left(\partial G / \partial g_{i}\right)^{-1}$ cannot decrease. Together, these observations show that the term in braces is 
strictly decreasing in $g_{i}$. Hence $\pi_{i}$ is a concave function of $g_{i}$, and the first order conditions are necessary and sufficient for best responses.

Using (3), these conditions can be written: $g_{i} \in\left(0, w_{i}\right]$ and

$$
M R S_{i}\left(G, w_{i}-g_{i}\right) \geq \frac{\rho G^{\rho-1}}{\beta_{i} \rho_{i}} g_{i}^{1-\rho_{i}} \text { with equality if } g_{i}<w_{i}
$$

Note that $\left(\partial G / \partial g_{i}\right)^{-1}=0$ at $g_{i}=0$, so this boundary value can never be a best response. For any $\mathbf{g}_{-i}$, define

$$
G_{-i}=\left(\sum_{j \neq i \beta_{i}} \beta_{j} g_{j}^{\rho_{j}}\right)^{1 / \rho}
$$

and observe that best responses to $\mathbf{g}_{-i}$ only depend on $G_{-i}$. We shall write $b_{i}\left(G_{-i}\right)$ for the best response to $\mathbf{g}_{-i}$ and recall that $b_{i}$ is a continuous function. In the case of Parameter Set 1 , it is possible to have $G_{-i}=0$, in which case $B R_{i}(0)$ maximizes $u_{i}\left(\beta_{i}^{1 / \rho} g_{i}^{\rho_{i} / \rho}, w_{i}-g_{i}\right)$ and therefore satisfies (4) with $G=\left(\beta_{i} g_{i}^{\rho_{i}}\right)^{1 / \rho}$. We put $\bar{G}_{i}=\left(\beta_{i}\left[B R_{i}(0)\right]^{\rho_{i}}\right)^{1 / \rho}$ and note that this is the level of the public good Player $i$ would provide if she were the sole contributor. Under Parameter Set 2, taking the limit of $g_{j} \longrightarrow \infty$ for all $j \neq i$ in (1) gives $G=\left(\beta_{i} g_{i}^{\rho_{i}}\right)^{1 / \rho}$ again and $\bar{G}_{i}$ is still the preferred level of the public good of Player $i$.

\subsubsection{Characterizing replacement functions}

It follows from Assumption A that, if we hold $G$ fixed, $M R S_{i}\left(G, w_{i}-g_{i}\right)$ is non-increasing in $g_{i}$. Furthermore, the right hand side of (4) is strictly increasing in $g_{i}$ and has zero slope at $g_{i}=0$ (since $\left.\rho_{i}<1\right)$. We can deduce that, for all $G>0$, (4) has a unique solution. We record this result below and illustrate it in Figure 2.

Lemma 5.2 Under increasing, binormal preferences and Parameter Set 1 or 2, there is a unique $g_{i} \in\left(0, w_{i}\right]$ satisfying (4). Furthermore, the feasibility condition $\beta_{i} g_{i}^{\rho_{i}} \leq G^{\rho}$ is satisfied for $G \geq \bar{G}_{i}$ under Parameter Set 1 and for $G \leq \bar{G}_{i}$ under Parameter Set 2 .

The proof of the second assertion is a little intricate and given in the appendix

We shall write $r_{i}(G)$ for the unique $g_{i}$ satisfying (4), where $r_{i}$ is the replacement function of Player $i$. The previous paragraph shows that $r_{i}$ is 


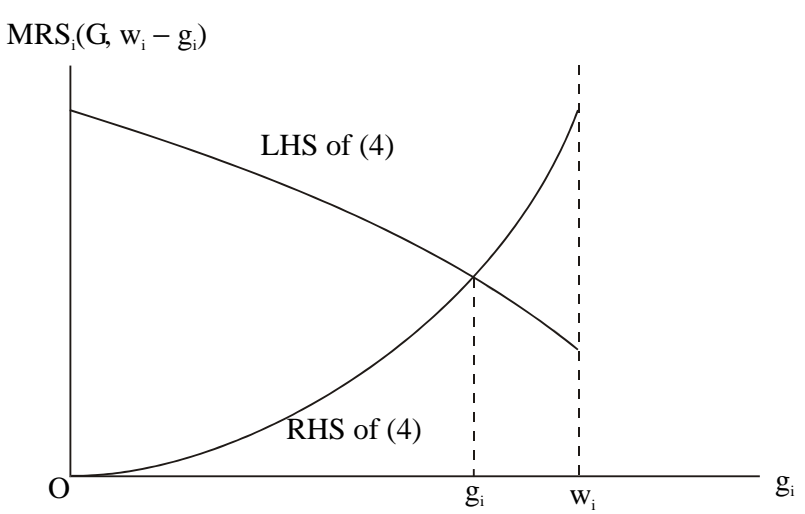

(a) Preferred $g_{i}<w_{i}$

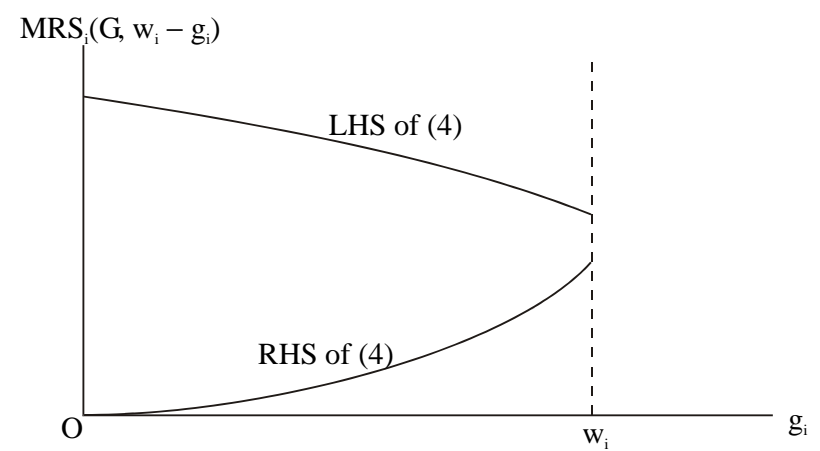

(b) Preferred $g_{\mathrm{i}}=\mathrm{w}_{\mathrm{i}}$

Figure 2:

defined for $G \geq \bar{G}_{i}$ under Parameter Set 1 and for $G<\bar{G}_{i}$ under Parameter Set 2 . The corresponding share function $s_{i}$ is defined as

$$
s_{i}(G)=\frac{\beta_{i}\left[r_{i}(G)\right]^{\rho_{i}}}{G^{\rho}}
$$

for all $G>0$ and we note that $\sum_{j=1}^{n} s_{j}(G)=1$ is a necessary and sufficient condition for positive $G$ to be a Nash equilibrium level of public good provision.

It is convenient, when studying the share function, to rewrite the interior first-order condition (4) with $\sigma_{i}=s_{i}(G)$ as

$$
\operatorname{MRS}_{i}\left(G, w_{i}-\beta_{i}^{-1 / \rho_{i}} \sigma_{i}^{1 / \rho_{i}} G^{\rho / \rho_{i}}\right)=\frac{\rho \beta_{i}^{-1 / \rho_{i}} \sigma_{i}^{\left(1-\rho_{i}\right) / \rho_{i}}}{\rho_{i}} G^{\left(\rho / \rho_{i}\right)-1} .
$$

Holding $\sigma_{i}$ fixed, an increase in $G$ leads to a strict decrease in $M R S_{i}$ and no decrease $G^{\left(\rho / \rho_{i}\right)-1}$ [under the assumptions of either parameter set.] Thus, 
the graph of the left-hand side of (6) shifts up, whereas the right-hand side shifts down or is unchanged. This is illustrated in Figure 3 and shows that $\sigma_{i}^{1 / \rho_{i}}$ decreases. Under Parameter Set 1 this means that $\sigma_{i}$ decreases, whereas,

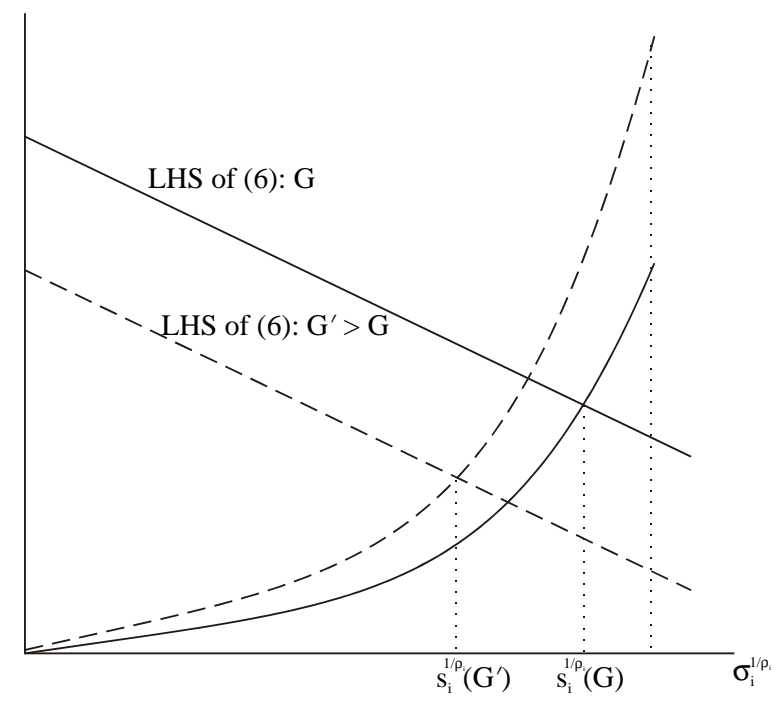

Figure 3:

under Parameter Set $2, \sigma_{i}$ increases.

Proposition 5.1 Under increasing, binormal preferences, the share function $s_{i}(G)$ of Player $i$ is continuous and strictly positive.

Under Parameter Set $1, s_{i}$ has domain $\left[\bar{G}_{i}, \infty\right)$, satisfies $s_{i}\left(\bar{G}_{i}\right)=1$, is strictly decreasing and has the limit $s_{i}(G) \longrightarrow 0$ as $G \longrightarrow \infty$.

Under Parameter Set 2, $s_{i}$ has domain $\left(0, \bar{G}_{i}\right)$, is strictly increasing and has the limits $s_{i}(G) \longrightarrow 0$ as $G \longrightarrow 0$ and $s_{i}(G) \longrightarrow 1$ as $G \longrightarrow \bar{G}_{i}$.

Continuity of $s_{i}$ follows from continuity of both $M R S_{i}$ and the right-hand side of (6). The limit $s_{i}(G) \longrightarrow 0$ under Parameter Set 1 is a consequence of the budget constraint: $r_{i}(G) \leq w_{i}$ for all $G$. The two possible forms for the share function are illustrated in Figure 4.

We can deduce from the proposition that, under Parameter Set 1, the aggregate share function $\sum_{j=1}^{n} s_{j}(G)$ is at least one if $G=\max _{j=1, \ldots, n}\left\{\bar{G}_{i}\right\}$, is continuous and strictly decreasing and approaches zero as $G \longrightarrow \infty$. It follows that the game has a unique equilibrium. Under Parameter Set 2, the aggregate share function is continuous and increases strictly from a value less that unity to a value greater than unity. Again, the game has a unique equilibrium. The following theorem records these results. 

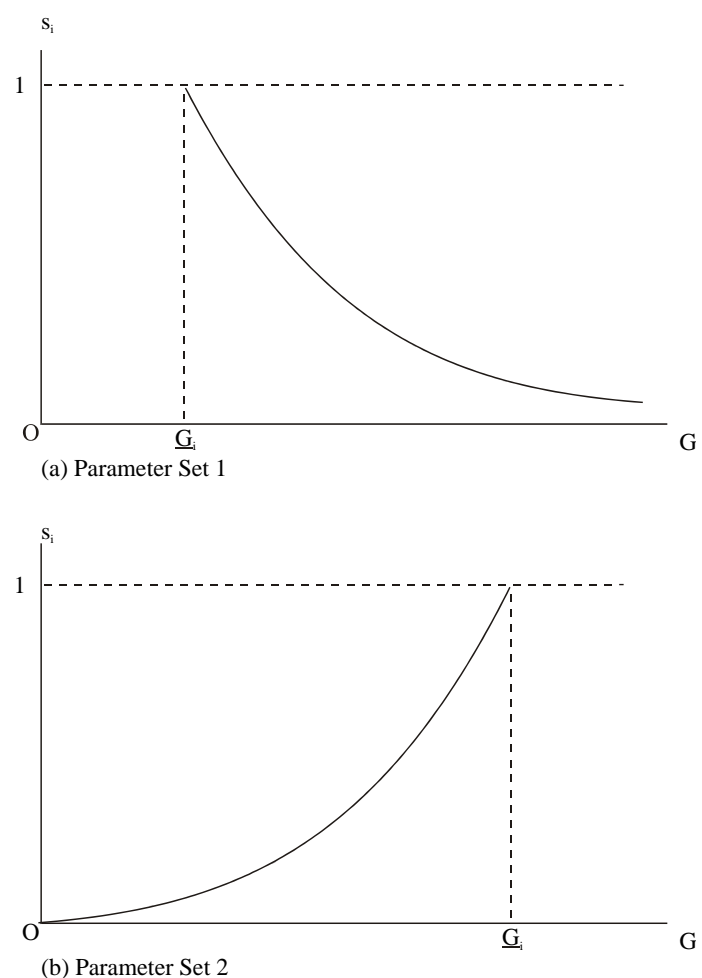

Figure 4:

Theorem 5.1 If all players have increasing, binormal preferences and the SCF (1) satisfies Parameter Set 1 or 2, there is a unique equilibrium.

\subsection{Cobb-Douglas preferences and the weakest-link limit}

In general it is not possible to derive analytical forms for replacement or share functions. This is true even for Cobb-Douglas preferences and a CES SCF. However, in this case we can find an explicit expression for the inverse of the replacement function. This enables us to examine the properties of the replacement function by studying the graph of this expression and reflecting it in the $45^{\circ}$ line. In this subsection, we illustrate this procedure and apply it to the study of the limit $\rho \longrightarrow-\infty$, and comparison of the weaker link and weakest link games. We shall assume that Player $i$ has utility function

$$
u_{i}\left(x_{i}, G\right)=x_{i}^{\alpha_{i}} G
$$


where $\alpha_{i}>0$ for all $i$ and that the SCF takes the CES form:

$$
G=\left(\sum_{j=1}^{n} \beta_{j} g_{j}^{\rho}\right)^{1 / \rho},
$$

for some $\rho<1$.

Since

$$
M R S_{i}\left(G, w_{i}-g_{i}\right)=\frac{w_{i}-g_{i}}{\alpha_{i} G}
$$

the first-order conditions (4) cannot be satisfied at $g_{i}=w_{i}$. Hence, all best responses are interior and the replacement function satisfies $r_{i}(G)=g_{i}$, where $g_{i}$ is the unique solution of

$$
G=\psi\left(g_{i}\right) \stackrel{\text { def }}{=}\left[\frac{\beta_{i}}{\alpha_{i}}\left(w_{i} g_{i}^{\rho-1}-g_{i}^{\rho}\right)\right]^{1 / \rho}
$$

and $G^{\rho} \geq \beta_{i} g_{i}^{\rho-1}$. That is, the graph of the replacement function is that portion of the reflection of the graph of $\psi$ satisfying $G^{\rho} \geq \beta_{i} g_{i}^{\rho-1}$. This is illustrated in Figure 5.

A CES SCF with all $\beta_{i}=1$ approaches the weakest-link SCF as $\rho \longrightarrow$ $-\infty$. Indeed, the CES SCF replacement function approaches the weakest link replacement function (pointwise) as the elasticity of substitution in the same limit. This follows by rewriting expression (7) as

$$
G=w_{i}\left(\frac{\beta_{i}}{\alpha_{i}}\right)^{1 / \rho}\left(1-\frac{g_{i}}{w_{i}}\right)^{1 / \rho}\left(\frac{g_{i}}{w_{i}}\right)^{1-1 / \rho}
$$

and, noting that $g_{i} / w_{i} \in(0,1)$, which implies that $G \longrightarrow g_{i}$ as $\rho \longrightarrow-\infty$. One might hope that the limit (if it exists) of the unique equilibrium of the weaker link game unambiguously selects the Pareto dominant profile from the continuum of equilibria found in the weakest link case. This need not be the case, however, as the following example illustrates.

Suppose there are $n \geq 2$ players and $\alpha_{i}=\alpha>0, w_{i}=w>0, \beta_{i}=1$ for all $i$. We shall write $r$ for the (common) replacement function of all players. Since the equilibrium is unique, it must be symmetric. This means that the equilibrium strategy profile is $\widehat{\mathrm{g}}=(\widehat{g}, \ldots, \widehat{g})$, where $\widehat{g}=r(\widehat{G})$ and $\widehat{G}^{\rho}=n \widehat{g}^{\rho}$. Substituting in (7), gives

$$
\widehat{g}=\frac{w}{\alpha n+1}
$$

and

$$
\widehat{G}=n^{1 / \rho} \frac{w}{\alpha n+1} \longrightarrow \frac{w}{\alpha n+1} \text { as } \rho \longrightarrow-\infty
$$



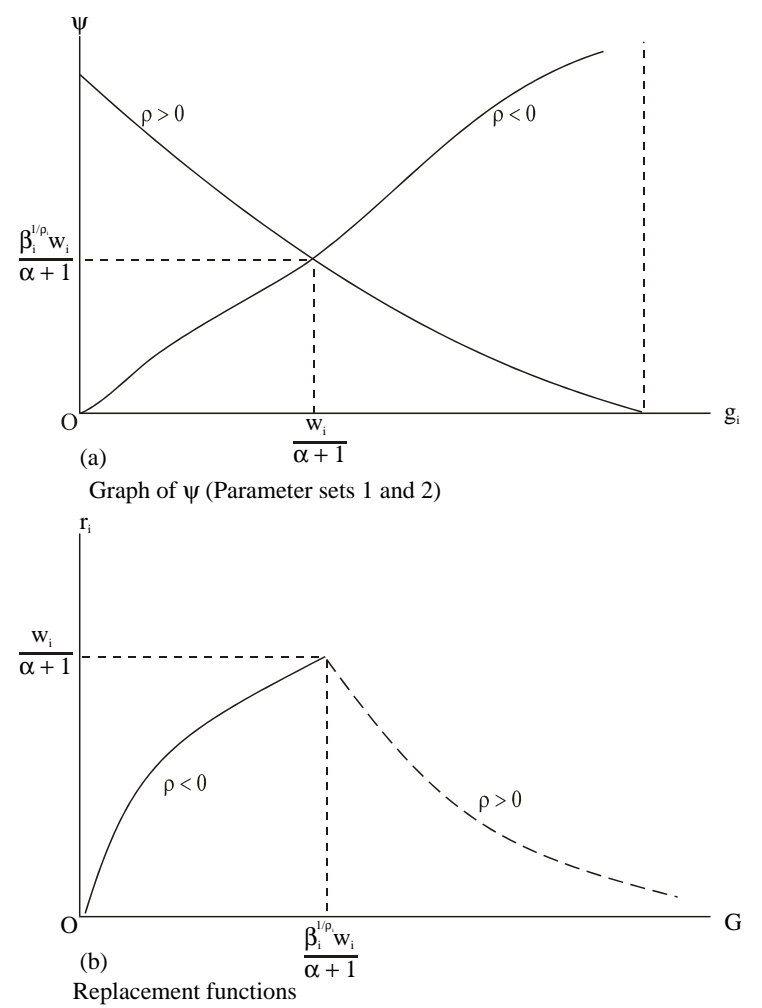

Figure 5:

Recall that $\bar{G}_{i}=g_{i}$, where $g_{i}$ maximizes $u_{i}\left(g_{i}, w_{i}-g_{i}\right)$. In the example, this gives

$$
\bar{G}_{i}=\frac{w}{\alpha+1} .
$$

The Pareto dominant equilibrium of the weakest link game has $g_{i}=\bar{G}_{i}$ for all $i$ and, since $n \geq 2$, this is not selected in the limit.

\section{Convex CES SCF}

\subsection{Best responses and the replacement correspondence}

When the SCF is not concave, even qualitative results are more sensitive to the specific form of the SCF and preferences. However, the approach of the preceding sections may still be adapted to handle such cases, provided we extend replacement and share functions to correspondences. To justify this 
claim, we focus on Cobb-Douglas preferences:

$$
u_{i}\left(x_{i}, G\right)=x_{i}^{\alpha_{i}} G,
$$

with $\alpha_{i}>0$ for all $i$ and CES SCF

$$
G=\left(\sum_{j=1}^{n} \beta_{j} g_{j}^{\rho}\right)^{1 / \rho}
$$

with $\rho>1$. In such a game, multiple equilibria are possible. However, provided $\rho$ is large enough, we can characterize sets of positive contributors and, given such an equilibrium set, show that the equilibrium is unique. For even larger $\rho$, all equilibrium sets consist of a single player. In the next section, we will show that, for such $\rho$, the equilibria coincide with those for the best-shot SCF.

Our approach uses a set-valued extension of replacement and share functions and we start by examining best responses. The payoff function of Player $i$ is

$$
\pi_{i}(\mathbf{g})=\left(w_{i}-g_{i}\right)^{\alpha_{i}} G,
$$

where $\mathbf{g}=\left(g_{1}, \ldots, g_{n}\right)$ and the strategy set is $\left[0, w_{i}\right]$. Writing

$$
G_{-i}=\left(\sum_{j \neq i} \beta_{j} g_{j}^{\rho}\right)^{1 / \rho}
$$

it is clear that the best response to $\mathbf{g}_{-i}$ actually depends only on $G_{-i}$ and we write $B_{i}\left(G_{-i}\right)$ for the set of best responses:

$$
B_{i}\left(G_{-i}\right)=\arg \max _{0 \leq g_{i} \leq w_{i}}\left\{\left(w_{i}-g_{i}\right)^{\alpha_{i}}\left(\beta_{i} g_{i}^{\rho}+G_{-i}^{\rho}\right)^{1 / \rho}\right\} .
$$

Intuitively, we might expect that, if the contributions of the other players are sufficiently large, a given player will free ride by contributing nothing. Indeed, we shall show that there is a critical value of $G_{-i}$ above which zero is the unique best response for Player $i$. Below this value, there is a single interior best response, which is a stationary point of $\pi_{i}$. At the critical value, both of the two strategies become alternative best responses.

Lemma 6.1 The following equations in $\left(g_{i}, G_{-i}\right)$ :

$$
\begin{aligned}
\beta_{i} w_{i} g_{i}^{\rho-1}-\beta_{i}\left(1+\alpha_{i}\right) g_{i}^{\rho} & =\alpha_{i} G_{-i}^{\rho}, \\
\left(w_{i}-g_{i}\right)^{\alpha_{i}}\left(\beta_{i} g_{i}^{\rho}+G_{-i}^{\rho}\right)^{1 / \rho} & =w_{i}^{\alpha_{i}} G_{-i}
\end{aligned}
$$


have a unique positive solution $g_{i}^{*}>0, G_{-i}^{*}>0$. The best response correspondence satisfies

$$
B_{i}\left(G_{-i}\right)= \begin{cases}\left\{b_{i}\left(G_{-i}\right)\right\} & \text { if } G_{-i}<G_{-i}^{*}, \\ \left\{0, b_{i}\left(G_{-i}\right)\right\} & \text { if } G_{-i}=G_{-i}^{*}, \\ \{0\} & \text { if } G_{-i}>G_{-i}^{*},\end{cases}
$$

where $b_{i}\left(G_{-i}\right)$ is the unique $g_{i} \in\left(0, w_{i}\right)$ satisfying

$$
\begin{aligned}
\alpha_{i}\left(\beta_{i} g_{i}^{\rho}+G_{-i}^{\rho}\right) & =\left(w_{i}-g_{i}\right) \beta_{i} g_{i}^{\rho-1}, \\
w_{i}^{\alpha_{i}} G_{-i} & \leq\left(w_{i}-g_{i}\right)^{\alpha_{i}}\left(\beta_{i} g_{i}^{\rho}+G_{-i}^{\rho}\right)^{1 / \rho} .
\end{aligned}
$$

The intuition behind this result is displayed in Figure 6. As a function of
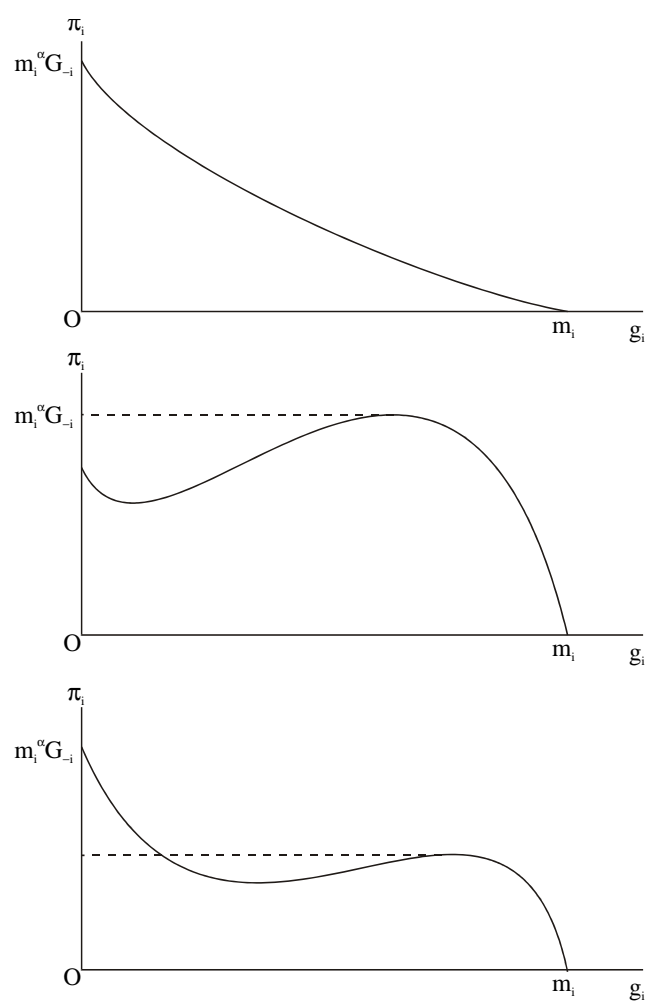

Figure 6:

own strategy, each player's payoff $\pi_{i}$ can take one of three forms. For large enough $G_{-i}$, the payoff decreases strictly as $g_{i}$ goes from 0 to $w_{i}$, (see first panel). For smaller $G_{-i}$, as $g_{i}$ increases from 0 to $w_{i}$, the payoff initially decreases, then increases to a local maximum and finally decreases to zero 
at $g_{i}=w_{i}$. The local maximum must be a stationary point: it satisfies (8). At $G_{-i}=G_{-i}^{*}$, the value of the payoff at the local maximum is the same as that at $g_{i}=0$. Equation (9) states this equality. For $G_{-i}<G_{-i}^{*}$, the payoff at the local maximum exceeds that at $g_{i}=0$ and the local maximum is therefore the global maximum (see second panel), whereas for $G_{-i}>G_{-i}^{*}$ the payoff is higher at $g_{i}=0$, which is the maximum in this case (see third panel.) Formal proof of these assertions may be found in the Appendix.

We define the replacement correspondence of Player $i$ to be $\mathcal{R}_{i}(G)$, where $g_{i} \in \mathcal{R}_{i}(G)$ if and only if $g_{i}$ is a best response to $G_{-i}$, where

$$
G_{-i}^{\rho}=G^{\rho}-\beta_{i} g_{i}^{\rho}
$$

Since $G=G_{-i}$ if $g_{i}=0$, the characterization of the replacement function becomes a corollary of Lemma 6.1.

Proposition 6.1 We have $g_{i} \in \mathcal{R}_{i}(G)$ if and only if (i) $g_{i}=0$ and $G \geq G_{-i}^{*}$ or (ii) $g_{i}>0$ and

$$
\begin{aligned}
\alpha_{i} G^{\rho} & =\beta_{i}\left(w_{i}-g_{i}\right) g_{i}^{\rho-1} \\
\beta_{i}^{1 / \rho} g_{i} & \leq G \leq \beta_{i}^{1 / \rho} w_{i}^{\alpha_{i}} g_{i}\left[w_{i}^{\alpha_{i} \rho}-\left(w_{i}-g_{i}\right)^{\alpha_{i} \rho}\right]^{-1 / \rho} .
\end{aligned}
$$

Equation (13) and the right-hand inequality in (14) are simply restatements of (10) and (11) using (12). The left-hand inequality is equivalent to the requirement $G_{-i} \geq 0$.

\subsection{Properties of the replacement and share correspon- dences}

In this subsection, we explore the properties of $\mathcal{R}_{i}$, noting that, if $g_{i} \in \mathcal{R}_{i}(G)$ and $g_{i}>0$, then $\left(g_{i}, G\right)$ must satisfy (13). Proposition 6.1 allows us to view the replacement correspondence as the reflection in the $45^{\circ}$ line of that portion of the graph of the function

$$
G=\left(\beta_{i} / \alpha_{i}\right)^{1 / \rho}\left[w_{i} g_{i}^{\rho-1}-g_{i}^{\rho}\right]^{1 / \rho},
$$

which also satisfies (14). Note that the right hand side of (15) vanishes at $g_{i}=0$ and $g_{i}=w_{i}$ and has a unique maximum at $g_{i}=(1-1 / \rho) w_{i}$. It is clear from Lemma 6.1, that the boundary line of the left-hand inequality in (14), $G=\beta_{i}^{1 / \rho} g_{i}$, crosses $(15)$ at $\left(\bar{g}_{i}, \bar{G}_{i}\right)$, where $\bar{g}_{i}=w_{i} /\left(1+\alpha_{i}\right)$ and $\bar{G}_{i}=\beta_{i}^{1 / \rho} \bar{g}_{i}$, whereas the boundary curve of the right-hand inequality in (14) crosses (15) to the left of this point, at $\left(g_{i}, G\right)=\left(g_{i}^{*}, G^{*}\right)$, where $G_{i}^{*}=$ $\left(\beta_{i} g_{i}^{* \rho}+G_{-i}^{* \rho}\right)^{1 / \rho}$. 

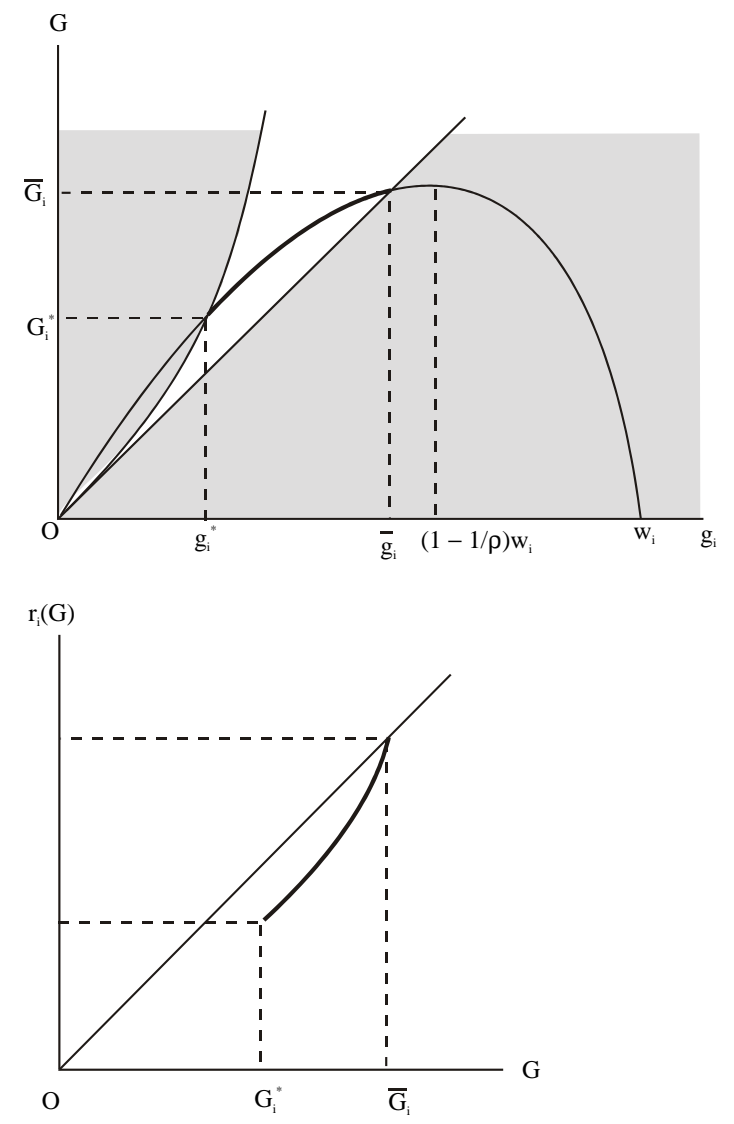

Figure 7: 
We shall initially analyze the case $\rho \geq 1+\left(1 / \alpha_{i}\right)$, for then both $\left(\bar{g}_{i}, \bar{G}_{i}\right)$ and $\left(g_{i}^{*}, G_{i}^{*}\right)$ lie to the left of (or at) the maximum of the function (15). This puts both points on the increasing portion of this function and implies that the positive portion of the replacement correspondence runs from $\bar{G}_{i}$ to $G_{i}^{*}$ and is increasing in this interval. This is the case illustrated in Figure 7

The share correspondence is defined as

$$
\mathcal{S}_{i}(G)=\left\{\frac{\beta_{i} g^{\rho}}{G^{\rho}}: g \in \mathcal{R}_{i}(G)\right\}
$$

and simple definition chasing verifies that $\widehat{G}$ is an equilibrium level of the public good if and only if there is $\widehat{\sigma}_{i} \in \mathcal{S}_{i}(\widehat{G})$ for all $i$ such that $\sum_{j=1}^{n} \widehat{\sigma}_{j}=1$. The associated equilibrium strategy profile is $\widehat{\mathbf{g}}$, where $\widehat{g}_{i}=\left(\widehat{\sigma}_{i} / \beta_{i}\right)^{1 / \rho} \widehat{G}$. Note that, if $\sigma_{i} \in \mathcal{S}_{i}(G)$ satisfies $\sigma_{i}>0$, equation (13) can be written

$$
\alpha_{i} G^{\rho}=\beta_{i}\left(w_{i}-\sigma_{i}^{1 / \rho} \beta_{i}^{-1 / \rho} G\right)\left(\sigma_{i}^{1 / \rho} \beta_{i}^{-1 / \rho} G\right)^{\rho-1} .
$$

This can be solved for $G$ as a function of $\sigma_{i}$ :

$$
G=\frac{\beta_{i}^{1 / \rho} w_{i} \sigma_{i}^{(\rho-1) / \rho}}{\alpha_{i}+\sigma_{i}}
$$

and this function has derivative

$$
\frac{\beta_{i}^{1 / \rho} w_{i} \sigma_{i}^{-1 / \rho}\left[\alpha_{i}(\rho-1)-\sigma_{i}\right]}{\rho\left(\alpha_{i}+\sigma_{i}\right)^{2}} .
$$

In the case that $\rho \geq 1+1 / \alpha_{i}$, this derivative is positive, since we also have $\sigma_{i}<1$. Since the function on the right-hand side of (16) is continuous and defined on a compact set, its inverse is also continuous. If we define $\sigma_{i}^{*}=\beta_{i} g_{i}^{* \rho} / G_{i}^{* \rho}$, the following proposition summarizes these observations and is illustrated in Figure 8.

Proposition 6.2 If $\rho \geq 1+1 / \alpha_{i}$, the graph of $\mathcal{S}_{i}$ is the disjoint union of two sets: $\left\{(G, 0): G \geq G_{-i}^{*}\right\}$ and $\left\{\left(G, s_{i}(G)\right): G_{i}^{*} \leq G \leq \bar{G}_{i}\right\}$. Furthermore, $s_{i}$ is continuous, strictly increasing and satisfies $s_{i}\left(G_{i}^{*}\right)=\sigma_{i}^{*}>0$ and $s_{i}\left(\bar{G}_{i}\right)=$ 1 .

Whenever the graph of a correspondence with domain a subset of the real numbers is the disjoint union of a finite family of connected sets, we refer to each of the correspondences whose graphs are these sets as components. When a component is singleton-valued, we also refer to the function defined 


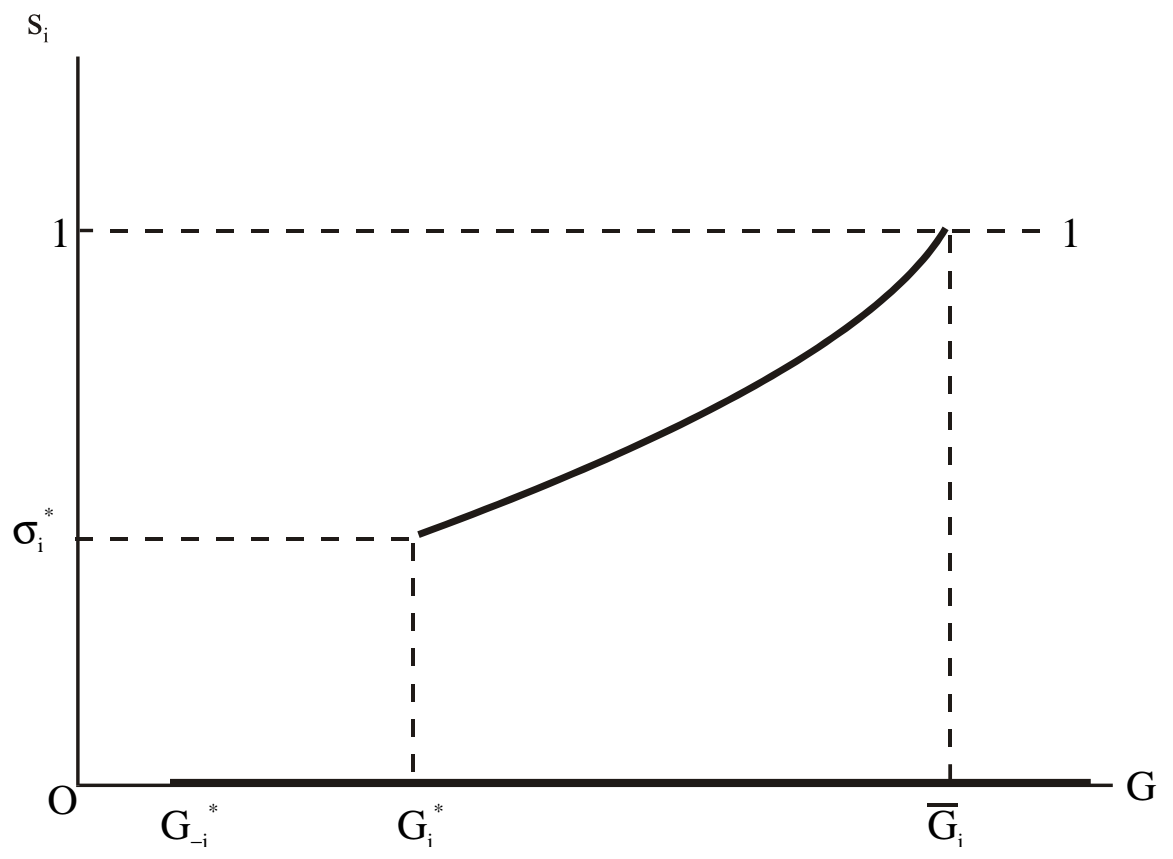

Figure 8:

by this correspondence as the component. The proposition says that $\mathcal{S}_{i}$ has two components: one is defined for $G \geq G_{-i}^{*}$ and is identically zero; the other is a continuous, strictly increasing function $s_{i}$ with domain $\left[G_{i}^{*}, \bar{G}_{i}\right]$.

In discussion of equilibria, it will prove convenient to refer to $s_{i}$ as the positive component of $\mathcal{S}_{i}$. Note that, if $\sigma_{i} \in s_{i}(G)$ satisfies $\sigma_{i}>0$, then $\sigma_{i} \geq \sigma_{i}^{*}$ and $G \geq G_{i}^{*}$. It follows that $g_{i} \geq\left(\sigma_{i}^{*}\right)^{1 / \rho} \beta_{i}^{-1 / \rho} G_{i}^{*}=g_{i}^{*}$.

Corollary 6.1 If $\rho \geq 1+1 / \alpha_{i}$ and player $i$ makes a positive contribution, that contribution is at least $g_{i}^{*}$.

When $\rho<1+1 / \alpha_{i}$, the portion of the graph of (15) satisfying (14) need not be increasing. Indeed, it may include increasing and decreasing sections or even have no increasing section ${ }^{5}$. Reflection in the $45^{\circ}$ line shows that the positive component of $\mathcal{S}_{i}$ may not be single valued, or it may be a decreasing function. Nevertheless, if $g \in \mathcal{R}_{i}(G)$ satisfies $g>0$, then $g \geq g_{i}^{*}$. Hence, Corollary 6.1 holds for all $\rho>1$. It can also be shown that $g_{i}^{*} \longrightarrow 0$ as $\rho \longrightarrow 1$. This shows that the disconnected nature of the replacement and share correspondences disappears as $\rho \longrightarrow 1$. Furthermore, it remains true

\footnotetext{
${ }^{5}$ In the latter case, the positive component would be strictly decreasing. It can be shown that this cannot happen if $\alpha_{i}<1$.
} 
that $\bar{g}_{i} \in \mathcal{R}_{i}\left(\bar{G}_{i}\right)$ and that $\mathcal{R}_{i}$ (and $\mathcal{S}_{i}$ ) has two components. We must modify Proposition 6.2 as follows.

Proposition 6.3 If $\rho<1+1 / \alpha_{i}$, the graph of $\mathcal{S}_{i}$ is the (disjoint) union of two sets: $\left\{(G, 0): G \geq G_{-i}^{*}\right\}$ and a connected set $S_{i}^{+}$which satisfies $\left(G_{i}^{*}, \sigma_{i}^{*}\right) \in$ $\mathcal{S}_{i}^{+},\left(\bar{G}_{i}, 1\right) \in \mathcal{S}_{i}^{+}$and $\sigma_{i}>0$ for all $\left(\sigma_{i}, G\right) \in \mathcal{S}_{i}^{+}$.

\subsection{Equilibria}

In this subsection, we characterize equilibria, starting with the case where $\rho \geq 1+1 / \alpha_{i}$ for all $i$. If we let $\underline{\alpha}=\min _{j=1, \ldots, n} \alpha_{j}$, this is equivalent to $\rho \geq 1+1 / \underline{\alpha}$. First note that the domain of the replacement correspondence of Player $i$ is $\left[G_{-i}^{*}, \infty\right)$. Hence, any equilibrium public good quantity must satisfy $G \geq \underline{G}=\max _{j=1, \ldots, n}\left\{G_{-j}^{*}\right\}$. For Player $i$ to be active (that is make a positive contribution), the equilibrium public good quantity must satisfy $G_{i}^{*} \leq G \leq \bar{G}_{i}$. If there is some equilibrium in which a player is active, we call that player potentially active. It follows from Proposition 6.2 that Player $i$ is potentially active if and only if $\bar{G}_{i} \geq \underline{G}$. If this inequality is not satisfied, we call Player $i$ always inactive. For each potentially active player, there is an equilibrium in which that player is the sole contributor, but there may also be equilibria with several contributors.

Theorem 6.1 Suppose that $\rho \geq 1+1 / \underline{\alpha}$ and $J$ is a non-empty set of potentially active players. There is a unique equilibrium with $J$ as the set of active players if and only if

$$
\begin{aligned}
G^{*}=\max _{j \in J}\left\{G_{j}^{*}\right\} & \leq \min _{j \in J}\left\{\bar{G}_{j}\right\}, \\
\sum_{j \in J} s_{j}\left(G^{*}\right) & \leq 1 .
\end{aligned}
$$

Furthermore, all equilibria are of this form.

The result is a corollary of Proposition 6.2. The first inequality is a consequence of the requirement that the equilibrium value $G$ must lie in the domain of $s_{j}$ and therefore satisfies $G_{j}^{*} \leq G \leq \bar{G}_{j}$ for all $j \in J$. The proposition also implies that $\sum_{j \in J} s_{j}(G)$ is a continuous, strictly increasing function satisfying

$$
\sum_{j \in J} s_{j}\left(\min _{k \in J}\left\{\bar{G}_{k}\right\}\right) \geq s_{k_{0}}\left(\bar{G}_{k_{0}}\right)=1,
$$


where $k_{0} \in \arg \min _{k \in J} \bar{G}_{k}$. Therefore $\sum_{j \in J} s_{j}(G)$ takes the value 1 if and only if (18) holds and, furthermore, does so for a single value of $G$. We refer to any set $J$ satisfying (17) and (18) as an equilibrium set and write $\widehat{G}(J)$ for the corresponding equilibrium value of $G$.

The share correspondences also determine the strategies played by the active players in an equilibrium set. Specifically, suppose $j$ is a player in equilibrium set $J$ and Player $j$ plays $\widehat{g}_{j}$ in equilibrium. Then,

$$
s_{j}(\widehat{G}(J))=\beta_{j} \widehat{g}_{j}^{\rho} \widehat{G}(J)^{-\rho},
$$

which implies that

$$
\widehat{g}_{j}=\left[s_{j}(\widehat{G}(J)) / \beta_{j}\right]^{1 / \rho} \widehat{G}(J) .
$$

Of course, $\widehat{g}_{k}=0$ for all $k \notin J$.

It is an immediate corollary of Theorem 6.1 that proper subsets of equilibrium sets are themselves equilibrium sets.

Corollary 6.2 Suppose that $\rho \geq 1+1 / \underline{\alpha}$, that $J$ is an equilibrium set that and non-empty $K$ satisfies $K \subset J$ (strict inclusion). If $\rho \geq 1+1 / \underline{\alpha}$, then $K$ is an equilibrium set and $\widehat{G}(K)>\widehat{G}(J)$.

Note that under the supposition of the corollary, if $J$ and $K$ are distinct equilibrium sets satisfying $K \subset J$, all players not in $K$ prefer this smaller equilibrium (as they free ride on a larger quantity of the public good.)

In a symmetric game, in which all players have the same preferences, we can be more precise in characterizing equilibrium sets. In such a game, the inequality (17) is superfluous and the inequality (18) is equivalent to $|J| \sigma^{*} \leq 1$, where $|J|$ denotes the cardinality of $J$ and $\sigma^{*}=\sigma_{i}^{*}$ for all $i$.

Corollary 6.3 If all players have the same preferences, a set of players is an equilibrium set if and only if it is non-empty and has at most $\left\lfloor 1 / \sigma^{*}\right\rfloor$ members, where $\lfloor x\rfloor$ denotes the integer part of $x$.

When $\rho<1+1 / \alpha_{i}$ for at least one $i$, we have seen that the positive component of the share correspondence need no longer be strictly increasing or even a function. This means that it is no longer possible to characterize equilibria as fully. The best that can be done is to give a sufficient condition related to Theorem 6.1. We need to extend our previous definition by saying that Player $i$ is potentially active if $\min \left\{\bar{G}_{i}, G_{i}^{*}\right\} \geq \underline{G}$. (Recall that, if $\rho \geq 1+1 / \alpha_{i}$, then $G_{i}^{*} \geq \bar{G}_{i}$, so this is just a generalization of our original definition.) The following result follows directly from Proposition 6.3 and continuity. 
Proposition 6.4 If $J$ is a non-empty set of potentially active players such that $\sum_{j \in J} \sigma_{j}{ }^{*} \leq 1$, there exists an equilibrium with $J$ as the set of active players.

It follows that, for each potentially active player, there is an equilibrium with that player as the sole contributor. However, we cannot conclude that subsets of equilibrium sets are also equilibrium sets, since the condition in the proposition is sufficient but not necessary.

\section{Best-shot Public Goods}

\subsection{Equilibria}

The best-shot SCF is

$$
G=\max _{j=1, \ldots, n} g_{j} .
$$

With such an SCF, replacement correspondences need not be well-defined. It is possible to have equilibrium strategy profiles $\widehat{\mathbf{g}} \neq \widehat{\mathbf{g}}^{\prime}$ satisfying $\sum_{j} \widehat{g}_{i}=$ $\sum_{j} \widehat{g}_{i}^{\prime}$. For example, suppose $\bar{G}_{i}$ denotes the preferred level of the public good of Player $i$ as sole contributor and $G_{-i}=\max _{j \neq i} g_{j}$. Then $g_{i}=\bar{G}_{i}$ is the best response to $G_{-i}=0$, whereas $g_{i}=0$ is the best response to $G_{-i}=\bar{G}_{i}$ and in both cases $G=\max \left\{g_{i}, G_{-i}\right\}=\bar{G}_{i}$. This means that there is no strategy that is a best response to all and every $G_{-i}$ satisfying $\max \left\{g_{i}, G_{-i}\right\}=G$. We can circumvent this difficulty by considering the upper replacement correspondence $\overline{\mathcal{R}}_{i}$ by putting $g_{i} \in \overline{\mathcal{R}}_{i}(G)$ if and only if there is some $G_{-i}$ such that $g_{i}$ is a best response to $G_{-i}$ and $\max \left\{g_{i}, G_{-i}\right\}=$ $G$. It is straightforward to establish that, if $\widehat{\mathrm{g}}$ is a Nash equilibrium, then

$$
\widehat{g}_{i} \in \overline{\mathcal{R}}_{i}(\widehat{G}) \text {, where } \widehat{G}=\sum_{j=1}^{n} \widehat{g}_{j} .
$$

In contrast to the case of replacement correspondences, if (19) holds, $\widehat{\mathrm{g}}$ need not be an equilibrium. Thus, the solutions of (19) provide a superset of the Nash equilibria and it is necessary to test each strategy profile in the superset to eliminate spurious members. In the particular case analyzed here, it turns out that there are no spurious members; (19) is necessary and sufficient for equilibrium $^{6}$.

To determine $\overline{\mathcal{R}}_{i}$, let $G_{-i}^{*}$ denote the level of $G_{-i}$ at which Player $i$ is indifferent between not contributing and being the sole contributor:

$$
\pi_{i}\left(0, G_{-i}^{*}\right)=\pi_{i}\left(\bar{G}_{i}, \bar{G}_{i}\right) .
$$

\footnotetext{
${ }^{6}$ This need not be true for general upper replacement correspondences.
} 
If $G_{-i}<G_{-i}^{*}$, Player $i$ will prefer to contribute $\bar{G}_{i}$ and $G=\bar{G}_{i}$. However, if $G_{-i}>G_{-i}^{*}$, Player $i$ is better off being a free rider and contributing nothing. In this case, $G=G_{-i}$. If $G_{-i}=G_{-i}^{*}$, the player is indifferent between the two strategies and $G=G_{-i}=G_{-i}^{*}$. This is clear from Figure 9. The form of the upper replacement correspondence is evident, described in the following proposition.

Proposition 7.1 If Player $i$ has convex, increasing preferences, her upper replacement function $\overline{\mathcal{R}}_{i}$ has domain $\left[G_{-i}^{*}, \infty\right)$ and satisfies

$$
\overline{\mathcal{R}}_{i}(G)= \begin{cases}\{0\} & \text { if } G \neq \bar{G}_{i}, \\ \left\{0, \bar{G}_{i}\right\} & \text { if } G=\bar{G}_{i},\end{cases}
$$

for all $G \geq G_{-i}^{*}$.

This is graphed in Figure 9.

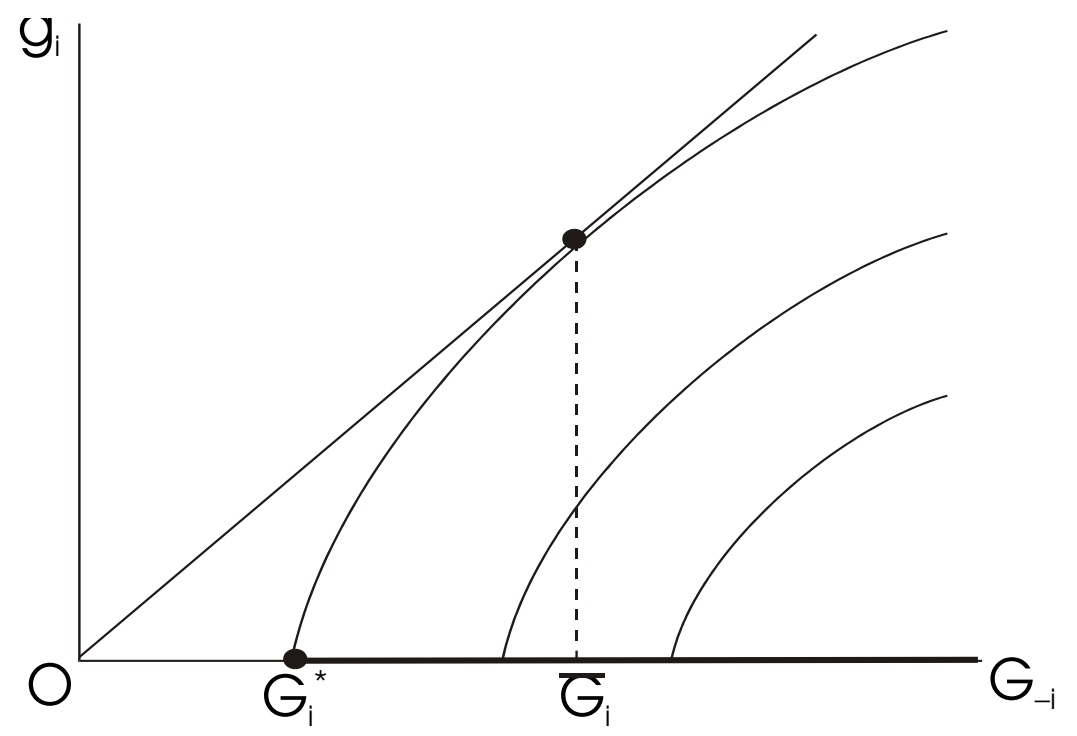

Figure 9:

To find equilibria, we look for solutions of (19) and observe first that $\widehat{G}$ must lie in the intersection of the domains of all $\overline{\mathcal{R}}_{i}$. From the proposition, we must have, $\widehat{G} \geq \max _{j} G_{-j}^{*}$. The proposition also implies that $\overline{\mathcal{R}}_{i}(G)=G$ for exactly one $i$. This implies $G=\bar{G}_{i}$. If we label every player $i$ for which $\bar{G}_{i} \geq \max _{j} G_{-j}^{*}$ as potentially active, every solution of (19) has $\widehat{g}_{i}=\bar{G}_{i}$ and 
$\widehat{g}_{j}=0$ for $j \neq i$ each potentially active player. It is trivial to verify that each such strategy profile is indeed a Nash equilibrium, so we have characterized the set of Nash equilibria.

Theorem 7.1 If all players have convex, increasing preferences there is a Nash equilibria are in 1-1 correspondence with the set of potentially active players. In each such equilibrium the potentially active player contributes her preferred level of the public good and no other player contributes.

Note that for best-shot games the theorem implies that (19) is necessary and sufficient for an equilibrium and therefore $\overline{\mathcal{R}}_{i}$ acts just like a replacement function.

\subsubsection{An example}

To illustrate the approach, consider two players with identical Cobb-Douglas preferences: $u_{i}=x_{i} G$ and incomes $w_{i}$ for $i=1,2$. Then, $\bar{G}_{i}=m_{i} / 2$ and $G_{-i}^{*}=m_{i} / 4$. If incomes do not differ too much, specifically $m_{2} \leq$ $2 m_{1} \leq 4 m_{2}$, both players are potentially active and there are two equilibria in each of which one player contributes half their income and the other free rides. If $m_{2}>2 m_{1}$, only Player 2 is potentially active and there is a unique equilibrium in which only Player 2 contributes $m_{2} / 2$. Similarly, if $m_{1}>2 m_{2}$, Player 1 is sole contributor. This raises the possibility of Pareto-improving transfers.

If $m_{1}=m_{2}=8$, there is an equilibrium $\widehat{\mathbf{g}}=(4,0)$ for which $\widehat{G}=4$ and payoffs are $\pi_{1}=16, \pi_{2}=32$, as well as an alternative equilibrium with Player 2 as sole contributor. If $m_{1}=4$ and $m_{2}=12$, there is a unique equilibrium: $\widehat{\mathrm{g}}=(0,6)$, in which $\widehat{G}=6$ and payoffs are $\pi_{1}=24, \pi_{2}=36$. It follows that, if Player 1 offers a transfer of 4 units of income to Player 2, the latter does better to accept and the former also benefits. Such a transfer also resolves the coordination problem arising from multiple equilibria. It can be shown further that, if $m_{2}<2 m_{1}<4 m_{2}$, a player anticipating an equilibrium in which they are sole contributor can find a transfer that results in a unique equilibrium with the other player as contributor in which both players have strictly greater payoffs than the anticipated equilibrium. Indeed, in some simple modifications of the two-player game with two equilibria, there will be transfers such that the post-transfer game has a unique equilibrium which strictly Pareto dominates both the equilibria in the original game. Clearly, the topic of transfers in best-shot games deserves further investigation. 


\subsection{Best-shot and better-shot games}

In this subsection, we return to the better-shot game of Section 6 and consider what happens as $\rho \longrightarrow \infty$. In this limit, the CES SCF approaches the bestshot SCF. The next result shows that the CES replacement correspondence approaches that of the best-shot SCF and does so monotonically. First, consider Player $i$ and note that $\left(G_{i}^{*}, g_{i}^{*}\right)$ satisfies (13) as well as equality in (14). Recall that preferences are Cobb-Douglas, $\bar{G}_{i}$ is the level of public good provision that Player $i$ would provide if she were the sole contributor and $g_{i}^{*}$ is the minimal contribution that Player $i$ will make in equilibrium.

Lemma 7.1 As $\rho$ increases, so does $g_{i}^{*}$ and $G_{i}^{*} \longrightarrow \bar{G}_{i}$ as $\rho \longrightarrow \infty$.

This shows that the positive component of the replacement correspondence shrinks to the tangency point of the $45^{\circ}$ line and the indifference curve in the $\left(G_{i}, g_{i}\right)$ plane. Thus Player $i$ 's replacement correspondence approaches the (upper) replacement correspondence of the best shot game. It follows that $\sigma_{i}^{*} \longrightarrow 1$ as $\rho \longrightarrow \infty$. Since $s_{i}(G) \geq \sigma_{i}^{*}$ for $G_{i}^{*} \leq G \leq \bar{G}_{i}$, the limit of the left hand side of (18) as $\rho \longrightarrow \infty$ exceeds 1 for any $J$ with two or more members and therefore no such $J$ can be an equilibrium set. All equilibrium sets must be singletons and are therefore equilibria of the best shot game.

Corollary 7.1 Under Cobb-Douglas preferences and CES SCF, there is a $\widehat{\rho}$ such that, if $\rho>\widehat{\rho}$, the set of equilibria coincides with that of the best-shot game.

Thus the best-shot game is more than just an approximation to the bettershot game. Equilibria of the latter can be analyzed by studying the (simpler) former game. It follows that the discussion of transfers in the previous subsection is also applicable to better-shot games.

\section{Conclusion}

The pure public good provision model of BBV is an outstandingly tractable model of reciprocal positive externalities. Its usefulness prompts one to enquire whether, and in what ways, its scope can be extended with minimal sacrifice of tractability. The present paper has explored extensions that modify the form of social composition function while retaining the game's aggregative structure. For reasons of space, we have concentrated on existence and uniqueness and limited ourselves to a few observations on comparative statics such as income redistribution. A more complete treatment of these 
issues is a subject for future research. Further extensions can also be envisaged that incorporate this aggregative structure - for example, the joint characteristics model of Cornes and Sandler may be revisited. We have explored circumstances under which our approach can be exploited to finesse what Richard Bellman once called, in another context, the "curse of dimensionality". The time seems ripe for further consideration of the range of interpretations and applications on which the model, and our method of analysis, may shed useful light. 


\section{APPENDIX}

In this appendix, we state and prove a lemma which is used in several other proofs. We then give several proofs displaced from the main text.

Lemma .1 For any $i=1, \ldots, n$ and $\rho>1$, there is a unique $x \in(0,1)$ satisfying

$$
\varphi_{i}(x ; \rho)=\left(1+\alpha_{i}\right) x+(1-x)^{\alpha_{i} \rho}=1 .
$$

Writing $\widetilde{x}(\rho)$ for this solution, $\widetilde{x}(\rho)$ is strictly increasing in $\rho$ and $\widetilde{x}(\rho) \longrightarrow$ $\left(1+\alpha_{i}\right)^{-1}$ as $\rho \longrightarrow \infty$.

Note that $x=0$ is always a solution of $(20)$, the lemma is concerned with positive solutions.

Proof. It is straightforward to check, using simple differentiation where appropriate, that

- $\varphi_{i}(0)=1$;

- $\varphi_{i}(1)=1+\alpha_{i}$;

- $\varphi_{i}$ is strictly decreasing ${ }^{7}$ for all small enough $x$;

- $\varphi_{i}$ is strictly convex.

This is illustrated in Figure 10. It follows that there is a unique $\widetilde{x}(\rho) \in$ $(0,1)$ such that $\varphi_{i}(\widetilde{x}(\rho) ; \rho)=1$ and that $\varphi_{i}(x ; \rho)$ is strictly increasing in $x$ in a neighborhood of $\widetilde{x}(\rho)$. Since $\varphi_{i}(x ; \rho)$ is strictly decreasing in $\rho$ for all $x \in(0,1)$, we may deduce that $\widetilde{x}(\rho)$ increases with $\rho$. Since $\widetilde{x}(\rho)<1$ for all $\rho>1$ there is $\bar{x} \in(0,1]$ such that $\widetilde{x}(\rho) \longrightarrow \bar{x}$ as $\rho \longrightarrow \infty$. Taking this limit in $(20)$ yields $\left(1+\alpha_{i}\right) \bar{x}=1$, completing the proof.

Proof of second assertion in Lemma 5.2. .We start by defining

$$
\widetilde{\gamma}_{i}\left(G_{-i}\right)=\left(\beta_{i}\left[b_{i}\left(G_{-i}\right)\right]^{\rho_{i}}+G_{-i}^{\rho}\right)^{1 / \rho}
$$

and observing that continuity of $b_{i}$ implies continuity of $\widetilde{\gamma}_{i}$. Note also that, if $g_{i}=r_{i}(G)$ and $G_{-i}^{\rho}=G^{\rho}-\beta_{i} g_{i}^{\rho_{i}}$, then $G=\widetilde{\gamma}_{i}\left(G_{-i}\right)$ In the case of Parameter Set 1, suppose that we had a $G^{\prime}$ satisfying (4) with $0<G^{\prime}<\bar{G}_{i}$. By Lemma 5.2, there is a unique $g_{i}^{\prime}$ satisfying (4) and this implies a unique $G_{-i}^{\prime}$ and $\widetilde{\gamma}_{i}\left(G_{-i}^{\prime}\right)=G^{\prime}<\bar{G}_{i}$. Now choose any $G_{-i}^{\prime \prime}>\bar{G}_{i}$ and note that $G^{\prime \prime}=$ $\widetilde{\gamma}_{i}\left(G_{-i}^{\prime \prime}\right) \geq G_{-i}^{\prime \prime}>\bar{G}_{i}$. By continuity, there would be a $G_{-i}^{\prime \prime \prime} \in\left(G_{-i}^{\prime}, G_{-i}^{\prime \prime}\right)$

\footnotetext{
${ }^{7}$ Its derivative approaches $-\alpha_{i}(\rho-1) m_{i}^{\alpha_{i} \rho}<0$ as $g_{i} \longrightarrow 0$.
} 


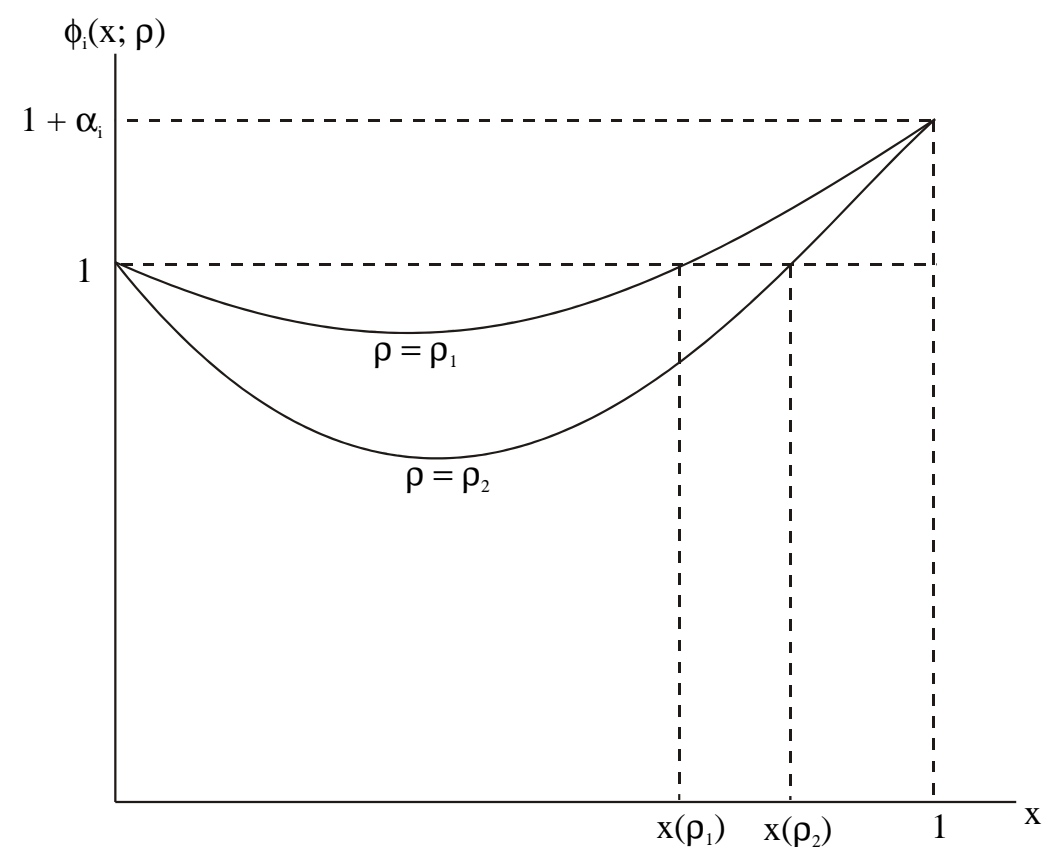

Figure 10:

such that $\widetilde{\gamma}_{i}\left(G_{-i}^{\prime \prime \prime}\right)=\bar{G}_{i}$ and $G_{-i}^{\prime \prime \prime}>0$ giving two distinct solutions to (4) with $G=\bar{G}_{i}$ and contradicting Lemma 5.2.

This argument requires some modification with Parameter Set 2. Suppose that $G^{\prime}>\bar{G}_{i}$, let $G_{-i}^{\prime}$ satisfy $\widetilde{\gamma}_{i}\left(G_{-i}^{\prime}\right)=G^{\prime}$ and note that, as $g_{j} \longrightarrow 0$ for some $j \neq i$, it follows from $(1)$ that $G \longrightarrow 0$. This means that we can choose $G_{-i}^{\prime \prime}$ such that $\widetilde{\gamma}_{i}\left(G_{-i}^{\prime \prime}\right)<\bar{G}_{i}$. We use the fact that, for any $\varepsilon>0$, there is a $G_{-i}^{\prime \prime \prime}>G_{-i}^{\prime \prime}$ and a $G^{\prime \prime \prime}$ within $\varepsilon$ of $\bar{G}_{i}$ such that (4) holds, which means that $\widetilde{\gamma}_{i}\left(G_{-i}^{\prime \prime \prime}\right)=G^{\prime \prime \prime}$. By continuity, there is a $G^{\prime \prime \prime \prime} \in\left(G_{-i}^{\prime}, G_{-i}^{\prime \prime}\right)$ such that $\widetilde{\gamma}_{i}\left(G_{-i}^{\prime \prime \prime}\right)=G^{\prime \prime \prime}$, giving two distinct solutions to (4) and contradicting Lemma 5.2.

Proof of Lemma 6.1. The payoff $\pi_{i}$ takes the value $w_{i}^{\alpha_{i}} G_{-i}$ at $g_{i}=0$ and zero at $g_{i}=w_{i}$. A little algebraic manipulation shows that stationary points of $\pi_{i}$ satisfy (8). Writing $\phi_{i}$ for the left-hand side of (8) it is straightforward to verify that $\phi_{i}(0)=0($ since $\rho>1)$ and $\phi_{i}\left(w_{i}\right)=-\beta_{i} \alpha_{i} w_{i}^{\rho}<0$. Further, $\phi_{i}$ has a unique stationary point $g_{i}^{\prime} \in\left(0, w_{i}\right)$ satisfying $\phi_{i}\left(g_{i}^{\prime}\right)>0$. We conclude that $g_{i}^{\prime}$ maximizes $\phi_{i}$ over $\left[0, w_{i}\right]$, which means that (8) has a solution if and only if $\alpha_{i} G_{-i}^{\rho} \leq \phi_{i}\left(g_{i}^{\prime}\right)$, so this is a necessary and sufficient condition for stationary points. Thus $\pi_{i}$ is a strictly decreasing in $\left[0, w_{i}\right]$ for $G_{-i} \geq \phi_{i}\left(g_{i}^{\prime}\right)$, has an interior local minimum and local maximum 
for $0<G_{-i}<\phi_{i}\left(g_{i}^{\prime}\right)$ and just an interior local (and global) maximum for $G_{-i}=0$. It follows that there are two cases.

A. A stationary point is the global maximum if the value of $\pi_{i}$ at that point is not exceeded by that at $g_{i}=0$; these two conditions are expressed in (10) and (11).

B. The global maximum is at $g_{i}=0$ if either $\alpha_{i} G_{-i}^{\rho} \geq \phi_{i}\left(g_{i}^{\prime}\right)$, or the value of $\pi_{i}$ at all stationary points does not exceed that at $g_{i}=0$.

The proof is completed by showing that $G_{-i}^{*}$ is well-defined and Case $\mathbf{A}$. holds for $G_{-i} \leq G_{-i}^{*}$ and Case B. for $G_{-i} \geq G_{-i}^{*}$. This can be achieved by showing that there is a unique $G_{-i}=G_{-i}^{*}$ for which both $\mathbf{A}$. and $\mathbf{B}$. hold and appealing to continuity ${ }^{8}$ together with the fact that Case $\mathbf{B}$. holds for large enough $G_{-i}$. Note that both $\mathbf{A}$. and $\mathbf{B}$. hold if and only if marginal payoff is zero and the payoff equals that at $g_{i}=0$; these conditions are equivalent to (8) and (9).

Raising (9) to the power $\rho$, multiplying by $\alpha_{i}$, substituting for $G_{-i}^{\rho}$ from (8) and dividing by $w_{i}^{\alpha_{i} \rho+1}$ gives $\varphi_{i}\left(g_{i} / w_{i} ; \rho\right)=1$, where $\varphi$ is defined in Lemma .1. Choose $g_{i}^{*}=w_{i} \widetilde{x}(\rho)$ and $G_{-i}^{*}$ to satisfy

$$
\alpha_{i} G_{-i}^{* \rho}=\beta_{i} w_{i} g_{i}^{* \rho-1}\left[1-\left(1+\alpha_{i}\right) \frac{g_{i}^{*}}{w_{i}}\right] .
$$

By construction, $\left(g_{i}^{*}, G_{-i}^{*}\right)$ satisfies (8) and (9). Lemma .1 implies that $0<\widetilde{x}(\rho)<\left(1+\alpha_{i}\right)^{-1}$ and therefore $g_{i}^{*}>0$ and $G_{-i}^{*}>0$.

Proof of Lemma 7.1. In the proof immediately above we showed that $\varphi_{i}\left[g_{i}^{*} / w_{i} ; \rho\right]=1$, where $\varphi$ is defined in Lemma .1. Recalling that $\bar{g}_{i}=w_{i}\left(1+\alpha_{i}\right)^{-1}$, this lemma implies that $g_{i}^{*} \uparrow \bar{G}_{i}$ as $\rho \longrightarrow \infty$.

\section{References}

[1] Arce M., D. G. and T. Sandler (2001), Transnational public goods: Strategies and institutions, European Journal of Political Economy, 17, 493-516.

[2] Bergstrom, T. C., Blume, L. and Varian, H. (1986), On the private provision of public goods, Journal of Public Economics, 29, 25 - 49.

[3] Bergstrom, T. C., Blume, L. and Varian, H. (1992), Uniqueness of Nash equilibrium in private provision of public goods: An improved proof, Journal of Public Economics, 49, 391-2.

\footnotetext{
${ }^{8}$ Alternatively, one can show that the payoff at $g_{i}=0$ increases faster than the payoff at any stationary point, using an envelope theorem.
} 
[4] Cornes, R. C. (1993), Dyke maintenance and other stories: Some neglected types of public goods, Quarterly Journal of Economics, 107, 259 $-71$.

[5] Cornes, R. C. and R. Hartley (2007), Aggregative public good games, Journal of Public Economic Theory, forthcoming.

[6] Fraser, C. D. (1992), The uniqueness of Nash equilibrium in the private provision of public goods: An alternative proof, Journal of Public Economics, 49, 389-90.

[7] Hirshleifer, J. (1983), From weakest-link to best-shot: the voluntary provision of public goods, Public Choice, 41, 371-86.

[8] Ihori, T. (1996), International public goods and contribution productivity differentials, Journal of Public Economics, 61, 139-54.

[9] Okuguchi, K. (1993), Unified approach to Cournot models: Oligopoly, taxation and aggregate provision of a pure public good, European Journal of Political Economy, 9, 233 - 45.

[10] Sandler, T. (1998), Global and regional public goods: A prognosis for collective action, Fiscal Studies, 19, 221-47.

[11] Sandler, T. (2003), Assessing the optimal provision of public goods: In search of the holy grail, in Kaul, I., P. Conceicao, K. le Goulven and R. U. Mendoza (eds.), Providing Public Goods: Managing Globalization, Oxford University Press: New York.

[12] Sandler, T. (2004), Global Collective Action, Cambridge University Press: New York.

[13] Sandler, T. and S. Vicary (2001), Weakest-link public goods: Giving in-kind or transferring money in a sequential game, Economics Letters, $74,71-5$.

[14] Spence, A. M. (1980), Notes on advertising, economies of scale, and entry barriers, Quarterly Journal of Economics, 94, 493-507.

[15] Varian, H. (2004), System reliability and free riding, unpublished manuscript.

[16] Vicary, S. (1990), Transfers and the weakest link: An extension of Hirshleifer's analysis, Journal of Public Economics, 43, 375-94. 
[17] Vicary, S. and T. Sandler (2002), Weakest-link public goods: Giving in-kind or transferring money, European Economic Review, 46, 1501-20. 